\title{
25 Research Soure \\ SARS-CoV-2 viral load predicts the severity and mortality in patients with cancer
}

\author{
Maha Al-Mozaini \\ King Faisal Specialist Hospital and Research Centre
}

Abu Shadat M Noman

Bangamata National Cellular and Molecular Research Centre

Jawaher Alotaibi

King Faisal Specialist Hospital and Research Centre

Mohammed Rezaul Karim

Park View Hospital

ASM Zahed

Park View Hospital

ATM Rezaul Karim

Park View Hospital

Khaldoun Alromiah

King Faisal Specialist Hospital and Research Centre

Syed S Islam ( $\square$ sislam83@kfshrc.edu.sa )

King Faisal Specialist Hospital and Research Centre

\section{Research Article}

Keywords: Cancer, chemotherapy, Covid-19, SARS-CoV-2, viral load, mechanical ventilation, intensive care unit, incubation period, serial interval

Posted Date: April 7th, 2021

DOI: https://doi.org/10.21203/rs.3.rs-400301/v1

License: (c) (i) This work is licensed under a Creative Commons Attribution 4.0 International License.

Read Full License 


\section{Abstract}

Emerging evidence suggests that patients with cancer are at increased risk of detrimental Covid-19 outcome. The relationship between SARS-CoV-2 viral load and risk factors and outcome of SARS-CoV-2 positive cancer patients remains largely unexplored. We assessed the outcomes of Covid-19 infection in 64 cancer patients and 120 non-cancer and measured SARS-CoV-2 viral load from nasopharyngeal swab samples using cycle threshold $(\mathrm{Ct}$ ) values who were admitted to two geographically distinct hospitals. We also assessed the incubation period and serial interval time differences between the non-cancer and cancer groups. Our results indicated that the overall mortality rate was higher among cancer patients with a high SARS-CoV-2 viral load. Covid-19 positive cancer patients with higher viral load are more prone to severe outcomes compared to non-cancer and low viral load patients. In addition, patients with lung and hematologic cancer have higher tendencies of severe events in proportion to high viral load. Higher attributable mortality and severity were directly proportional to high viral load particularly patients who are receiving anticancer treatment. Importantly, we found that the incubation period and serial interval time is fairly shorter in cancer patients compared with non-cancer cases. Our report suggests that high SARS-CoV-2 viral loads may play significant role in the overall mortality and severity of Covid-19 positive cancer patients and warranted further study to explore the disease pathogenesis and their use as prognostic tools.

\section{Introduction}

Individuals who are infected with severe acute respiratory syndrome coronavirus 2 (SARS-CoV-2) are phenotypically diverse depending on the history of active malignancies. The Covid-19 symptoms ranging from mild to severe and may have an extreme outcome. Patients with cancer and Covid-19 have been identified as increased risk of mortality and morbidity ${ }^{1}$.

Recent reports suggest that age, male sex, smoking status and comorbidities (hypertension, cardiovascular disease and diabetes) have been widely identified as risk factors and severely impact on patients mortality in patients with cancer ${ }^{2,3}$. Among other comorbid factors, cancer was identified as a susceptible group and have been identified as an increased risk of infection with Covid-19 and increased risk of death and severe outcomes. These severe phenotypes require special attention with higher intensive care, rapidly deteriorating patient conditions and increased risk of death ${ }^{4-6}$ with a clear contrast between the patients with and without cancer. Furthermore, the clinical phenotypes of patients with cancer and the effects of anticancer treatment greatly influence the outcome of the patient's severity and survival.

It was reported that high SARS-CoV-2 viral load was independently associated with in-hospital mortality among Covid-19 positive population ${ }^{7}$. In addition, high SARS-CoV-2 viral load in patients with cancer have been reported recently ${ }^{8,9}$ Although this report highlighted the relationship between viral loads and mortality of cancer and non-cancer patients, the impact of viral loads on outcomes of patients with 
cancer with a specific cancer type and anti-cancer treatment require additional study to determine whether intensity of SARS-CoV-2 viral load may precisely predict the outcome of cancer patients.

We aimed to illustrate the clinical characteristics and outcome of patients with and without cancer and presented evidence the detrimental effects of SARS-CoV-2 viral loads among patients with and without cancer. Here we show that SARS-CoV-2 high viral loads adversely affect the certain type of cancer patients particularly who are under chemotherapeutic treatment which may provide predictive tool for cancer patients mortality.

\section{Methods}

\section{Study design and patients}

In this study, we collected data from a primary (Bangladesh) and a tertiary (Riyadh) health care centers among patients with Covid-19 who have been diagnosed with cancer recently or in the past. All cancer patients enrolled in this study were confirmed with Covid-19 who were admitted to the hospitals from June 30, 2020 to August 7, 2020. A control cohort without cancer with confirmed Covid-19 infection who were admitted to same hospitals during the same time period were collected. The cancer cohort comprised 64 cases, while the non-cancer cohort comprised 120 cases. We excluded patients who displayed radiological or clinical diagnosis of Covid-19, but without a positive RT-PCR results. For cancer cohort, patients with active cancer were defined as those undergoing anticancer treatment with curative, radical, adjuvant or neoadjuvant therapy or treated in the last 12 months with radiotherapy, surgery, chemotherapy. Both datasets contained mainly information on exposure time, time of symptoms onset, as such all cancer and non-cancer cases, by definition are symptomatic. Four clinical outcomes were monitored up to August 30, 2020. We have classified disease severity in 4 main categories. (a) "mild cases" if the patients do not show any serious symptoms described in moderate, severe and deceased categories but positive nasopharyngeal swab RT-PCR test and did not require serious medical intervention; (b) "moderate cases" if the patients show fever, some respiratory symptoms and any evidence of pneumonia by radiography; (c) "severe cases" defined as cases experiencing any of the following symptoms: breathing rate 30 or higher breaths/minute, the oxygen saturation level below 93\%, one or multiple organ failures, requiring intensive care unit (ICU) and invasive mechanical ventilation support (IVS), a ratio of the partial pressure of arterial oxygen to the fraction of inspired oxygen (PaO2:FiO2) of less than $300 \mathrm{~mm} \mathrm{Hg}$, or infiltrates in more than $50 \%$ of the lung field within 24 to 48 hours; and (d) "deceased cases" patients admitted to the hospitals with Covid-19 related symptoms and died during their hospital stay. This study was considered exempt from the requirements of institutional review board (IRB) approval and was approved by the central ethics committee of Bangladesh Medical Research Council (study \#2021-2023/62(1-20) and King Faisal Specialist Hospital and Research Centre (RAC \# 2200031).

\section{SARS-CoV-2 Viral RNA load measurements}


Nasopharyngeal swab samples were collected from cancer and non-cancer patients and samples were stored in room temperature and processed within reasonable time of collection. Nucleic acid was extracted from samples using MOLgen-SARS-CoV-2 and Sansure Biotech SARS-CoV-2 assay protocol according to the manufacturer's instructions. Extracted RNA was eluted using elusion buffer. Levels of SARS-CoV-2 viral load was determined using US CDC real-time RT-PCR primer and probe set for 2019nCoV N1 and N2. Ct values obtained from the N1 primers set were converted to RNA copies/mL using N1 quantitative PCR (qPCR) in 10-fold dilution standard curve as described previously 10,11 . Values were $\log _{10}$ transformed. We converted Ct values into quantitative assessments of viral load- high, Ct value < 21 , medium, Ct value 21-26, and low, Ct value $>26$. These cutoff values (high, medium and low) were used to determine the risk of severity and death of all patients used in this study.

\section{Data collection and procedures}

For this study, we collected data from patients by our data collection team with the assistance participating hospitals registry personnel. Patients demographics, cancer details, cancer treatment information, age, sex, number of comorbidities which require active treatment, surgical history and diagnosis of Covid-19, cancer status, treatment during hospitalization were collected.

\section{Outcomes}

We considered and analyzed four clinical outcome/endpoints: severe illness requiring admission to the hospital, death, admission to ICU, needing invasive mechanical ventilation support (IVS).

\section{Incubation period analysis}

We recorded daily mortality and hospitalization incidence and plotted with cumulative numbers of confirmed and discharged cases based on symptom onset date for non-cancer and cancer cohorts. We excluded cases that do not have definitive symptom onset date. We generated a source of disease plot based on the best information available during the study period. For incubation period analysis, we used direct estimation methods ${ }^{12}$ based on the earliest and latest possible exposure times and reported symptom onset times. The parametric distributions (gamma distribution, Weibull distribution, and lognormal distribution) of incubation period was estimated using interval censoring using maximum likelihood of a time falling in a defined window. We used interval censoring because it is not possible to know the exact time of exposure.

\section{Serial interval analysis}

We estimated serial intervals in a direct method as the difference between the symptom onset dates. We excluded asymptomatic cases as well those cases that do not have exact symptom onset dates. We fitted the normal, log normal, gamma and Weibull distributions using R packages "fitdstrplus" by maximum-likelihood of a time falling in a defined window. We computed each fit distribution using 
Akaike information criteria (AIC) scores and calculated confidence intervals for parameters from 1000 bootstrap replicates ${ }^{13}$.

\section{Statistical analysis and data visualization}

All statistical analyses were performed using R (R Development Core Team, 2013). We assessed and reported clinical outcomes of Covid-19 positive non-cancer and cancer patients whether patients died or discharged and the effects of anti-cancer therapy on patients underlying conditions. We calculated the percentages of patients in each category for the categorical data. The Wilcoxon rank sum test was used for continuous data, and two-sided Fisher's exact test was used to compare categorical data for different categories of data without multi-test. Multivariate logistic regression analysis was used to estimate the odd ratio (OR) and $95 \%$ confidence interval $(\mathrm{Cl})$ of each factor of interest with confounders/outcomes. The OR was adjusted to chronic renal disease, cardiac disease, diabetes, hypertension, asthma and pulmonary disease. A two-sided $P$ values $<0.05$ was used to indicate the statistical significance. We constructed a multivariate logistic analysis model to identify variables that were independently associated with high and low viral load. Correlation analysis was performed using Spearman-rank based testing.

\section{Results}

\section{Clinical characteristics of patients among non-cancer and cancer patients}

We have obtained and analyzed 64 RT-PCR confirmed COVID-19 positive cancer patients from June 30, 2020 , to August 7, 2020 from a primary care hospital in Bangladesh and a tertiary care hospital King Faisal Specialist Hospital and Research Centre, Riyadh. In addition, we have collected 120 COVID-19 positive patients without the history of cancer from the same hospitals. The latter group was used as a control group to compare the parameters between cancer and non-cancer patients. The median age of cancer patients was 55 (inter quartile range [IQR)-10.5], and 53 (IQR 9.0) for non-cancer patients ( $p<$ 0.0001; Table 1). In our patient population age distribution range for cancer and non-cancer were close. Figure $1 \mathrm{~A}$ shows the age distribution of patients in non-cancer and cancer patients. In total, the two cohort comprises with 123 males and 61 females' cases and male female ratio is 2:1 (Table 1). The source of infection mostly occurred from hospital 41 (34.1\%) out of 120 and 29 (45.31\%) out of 64 for non-cancer and cancer cases respectively. When assessing the patient's comorbid factors, diabetes ranks the top (31/120[25.83\%] vs 13/64 [20.31\%) for non-cancer cases. However, hypertensive condition was deadliest in cancer cases (19/64 [29.69\%] vs 21/120 [17.5\%]) than non-cancer cases. Typical Covid-19 related signs and symptoms fever (65/120 [54.17\%] vs 29/64 [45.31\%]) and cough (43/120 [35.83\%] vs $26 / 6440.63 \%]$ ) was the highest in both cancer and non-cancer cases. Table 1 summarizes the patients clinical characteristics. 
Table 1

Characteristics of Covid-19 positive non-cancer and cancer patient

\begin{tabular}{|c|c|c|c|}
\hline Characteristics & $\begin{array}{l}\text { Covid-19 patients without } \\
\text { cancer }(n=120)\end{array}$ & $\begin{array}{l}\text { Covid-19 patient with } \\
\text { cancer }(n=64)\end{array}$ & $\begin{array}{l}p- \\
\text { values }\end{array}$ \\
\hline Age (years, median/IQR & $53.0 / 9.0$ & $55 / 10.5$ & $\begin{array}{l}< \\
0.0001\end{array}$ \\
\hline \multicolumn{4}{|l|}{ Sex } \\
\hline Male & $75 / 62.5 \%$ & $48 / 75.0 \%$ & 0.63 \\
\hline Female & $45 / 37.5 \%$ & $16 / 25 \%$ & 0.59 \\
\hline \multicolumn{4}{|l|}{ Source of infection } \\
\hline Hospital & $41 / 34.17 \%$ & $29 / 45.31 \%$ & 0.16 \\
\hline Family & $14 / 11.67 \%$ & $15 / 23.44 \%$ & 0.77 \\
\hline Unknown & $65 / 54.17 \%$ & $20 / 31.25 \%$ & 0.15 \\
\hline \multicolumn{4}{|c|}{ Underlying medical conditions } \\
\hline Chronic renal disease & $9 / 7.5 \%$ & $3 / 4.69 \%$ & 0.69 \\
\hline Cardiac disease & $11 / 9.17 \%$ & $15 / 23.44 \%$ & 0.003 \\
\hline Diabetes & $31 / 25.83 \%$ & $13 / 20.31 \%$ & 1.0 \\
\hline Hypertension & $21 / 17.5 \%$ & $19 / 29.69 \%$ & 0.05 \\
\hline Asthma & $5 / 4.17 \%$ & $1 / 1.56 \%$ & 1.0 \\
\hline Pulmonary disease & $4 / 3.33 \%$ & $1 / 1.56 \%$ & 1.0 \\
\hline No known disorders & $39 / 32.5 \%$ & $12 / 18.75 \%$ & 0.07 \\
\hline \multicolumn{4}{|l|}{ Hospitalization status } \\
\hline Discharged & $109 / 90.33 \%$ & $45 / 70.31 \%$ & 0.56 \\
\hline Deceased & $9 / 7.5 \%$ & $19 / 29.69 \%$ & 0.04 \\
\hline $\begin{array}{l}\text { Duration in hospital } \\
\text { stay (days) }\end{array}$ & 13.6 & 23.8 & 0.0001 \\
\hline \multicolumn{4}{|c|}{ Typical Covid-19 signs and symptoms } \\
\hline Fever & $65 / 54.17 \%$ & $29 / 45.31 \%$ & 0.03 \\
\hline Cough & $43 / 35.83 \%$ & $26 / 40.63 \%$ & 0.02 \\
\hline Nausea & $3 / 2.5 \%$ & $6 / 9.34 \%$ & 0.07 \\
\hline Fatigue & $2 / 1.67 \%$ & $3 / 4.69 \%$ & 0.08 \\
\hline
\end{tabular}




\begin{tabular}{|llll|}
\hline Characteristics & $\begin{array}{l}\text { Covid-19 patients without } \\
\text { cancer }(\boldsymbol{n}=\mathbf{1 2 0})\end{array}$ & $\begin{array}{l}\text { Covid-19 patient with } \\
\text { cancer }(\mathbf{n}=64)\end{array}$ & $\begin{array}{c}\text { p- } \\
\text { values }\end{array}$ \\
\hline Sore throat & $7 / 5.83 \%$ & $2 / 3.13 \%$ & 1.0 \\
\hline Treatments during hospitalization & & 0.39 \\
\hline Antiviral treatment & $49 / 40.83 \%$ & $19 / 29.61 \%$ & 0.0001 \\
\hline Oxygen therapy & $19 / 15.83 \%$ & $23 / 35.94 \%$ & 0.04 \\
\hline Antibiotic treatment & $46 / 38.33 \%$ & $25 / 39.06 \%$ & 0.04 \\
\hline Invasive mechanical & $6 / 5.0 \%$ & $13 / 20.31 \%$ & \\
\hline ventilation & & & N/A \\
\hline Cancer type & N/A & $16 / 25.0 \%$ & N/A \\
\hline Breast cancer & N/A & $5 / 7.81 \%$ & N/A \\
\hline Ovary & N/A & $9 / 14.06 \%$ & N/A \\
\hline Lung/NSCLC & N/A & $3 / 4.69 \%$ & N/A \\
\hline Hematologic cancer & N/A & $6 / 9.38 \%$ & N/A \\
\hline Bladder & N/A & $7 / 10.94 \%$ & N/A \\
\hline Colon & N/A & $6 / 9.38 \%$ & N/A \\
\hline HNSCC & N/A & $5 / 7.81 \%$ & $3 / 4.69 \%$ \\
\hline Rectal & N/A & $4 / 6.25 \%$ & \\
\hline Pancreas & N/A & & \\
\hline Esophageal & & & \\
\hline
\end{tabular}

Overall, intensive care unit (ICU) admission, disease severity and invasive ventilation support requirements was urgently needed for cancer patients compared to non-cancer patients (Fig. 1B) and the death was principally attributed to Covid- 19 positive cancer patients. In the cancer cohort, the death rate was strikingly higher $(29.69 \%)$ compared to non-cancer cases $(7.5 \%$, Fig. $1 \mathrm{~B}, \mathrm{p}<0.05)$ given the fact of similar age distribution between cancer and non-cancer patients. The requirement of ICU admission was greater $(20.31 \%, p=0.01)$ in cancer patients than non-cancer cohort, higher rate of having severe condition (37.5\%; $p=0.05)$, and needing and utilizing invasive ventilation support (IVS; $12.5 \% ; p=0.01$; Fig. 1B).

As of August 15, 2020, 28 (10.32\%) patients had died in the non-cancer (9/120; 7.5\%) and cancer (19/64; $29.69 \%$ ) cohort, all within 15 days with reports stating that the death was substantially attributed to Covid-19 in all patients. The patients who died from Covid-19 related complications in the cancer and 
non-cancer patients were mostly older age group. The age distribution of death rate in cancer patients was between 40-59 years, while for non-cancer patients it was between 40-69 years of age

(Supplementary Fig.S1A). We also stratified the severe condition of all cancer and non-cancer patients by age group. In general, the requirement of ICU support for cancer patient was higher in the older age group (40-69 years), but in the non-cancer group only patients age between 60-79 needed for ICU support requirement (Supplementary Fig. S1B). The symptoms of severity in cancer and non-cancer patients increased with the increase of age (Supplementary Fig. S1C), and ventilation support was proportionally higher in cancer patients age group between 40-69 years (Supplementary Fig. S1D). In contrast, no invasive ventilation support was needed for non-cancer patients at any given age group (Supplementary Fig. S1D).

The overall pooled odd ratio (OR) of all identified comorbidity for non-cancer patient was 0.45 (95\% Cl: 0.240 .86 , Supplementary Fig. 1E). A forest plot of the potential underlying conditions is shown in Supplementary Figure S1E. Covid-19 positive non-cancer patients with cardiac disease (odd ratio: 0.33, 95\% Cl: $0.09-1.27$ ), diabetes (odd ratio: $0.62,95 \% \mathrm{Cl} 0.16-2.43$ ), and hypertension (odd ratio: $0.82,95 \%$ $\mathrm{Cl} 0.28-2.42$ ) remained the deadliest comorbidity factors among all non-cancer patients (Supplementary Fig. S1E). According to multivariate regression analysis, Covid-19 positive cancer patients had overall higher OR (OR, 2.59; 95\% Cl 1.47-5.26; $\mathrm{p}<0.001$; Supplementary Table S1a) for death, ICU admission (OR 4.22; 95\% Cl 3.17-6.26, $\mathrm{p}<0.001$; Supplementary Table S1b) and severe symptoms (OR 2.26; $95 \% \mathrm{Cl}$ 1.77-4.61, $\mathrm{p}<0.01$; Supplementary Table S1c) when considering with other underlying conditions.

\section{Incubation and serial interval period analysis for non-cancer and cancer patients}

Types of malignancies in the Covid-19 patients may greatly influence the patient's outcomes. To expand our understanding of patient's malignancies and symptom onset between cancer and non-cancer patients, we have investigated the incubation period and serial interval time. Our study composed of several cancer types where breast cancer was overrepresented $(16 / 64,25.0 \%)$, followed by lung $(9 / 64,14.06 \%)$, colon $(7 / 64,10.94 \%)$ and head and neck cancer $(6 / 64,9.38 \%)$ (Fig. 2A). The overall death rate in cancer patients was $29.69 \%$. The death rate among lung cancer patients was higher $(7 / 64$, $10.93 \%)$, while hematologic and ovarian cancer patients had the second highest death rate $(3 / 64,4.69 \%$; $3 / 64,4.69 \%)$. Breast, colon, and gastrointestinal cancer each had 2 deaths out of $64(2.94 \%)$ (Supplementary table S2). The patients who had a high death rate also accompanied with severe and critical symptoms, longer ICU supports and invasive mechanical ventilation requirements (Supplementary Table S2).

Many recently published manuscripts reported that the mean incubation period for Covid-19 positive cases was between 5.6 to 6.7 days and the pooled parameter estimates the median period of 5.1 and 11.7 days ${ }^{14}$. In the non-cancer cohort, we find that the median incubation period is 5.41 days with the gamma distribution: shape 4.84 (95\% Cl: $3.41-5.93)$ and scale 1.2 (95\% Cl 0.81-1.34). On the other hand, the mean incubation period is 5.81 (95\% Cl: 5.17-6.50). In the cancer cohort, the median incubation period is 4.10 days with the gamma distribution: shape is 1.89 ( $95 \% \mathrm{Cl}$ : 1.32-2.42) and scale 
2.61 (95\% Cl: $1.47-3.79)$. The calculated mean is 4.99 (95\% Cl: $3.60-6.71)$ days. Summary of the results is presented in supplementary table 33 and fitted Weibull and log normal distributions is in supplementary table $\mathrm{s} 4$. Although our results are consistent with recently published data for non-cancer group, while the cancer patient's incubation time was shorter than the non-cancer counterparts. These results suggest that the estimated mean incubation period for cancer patients is likely shorter than the non-cancer Covid-19 positive patients. Supplementary table $s 5$ shows the recently published estimated mean incubation period and serial interval for Covid-19 positive non-cancer patients. A heatmap presentation of disease progression in Figs. $2 \mathrm{~B}$ and $2 \mathrm{C}$ for 120 non-cancer and 64 cancer cases shows that Covid-19 related symptom was 3.48+/-2.17 (mean+/-sd) days after first possible exposure to virus and most cases were confirmed 2.43+/-1.47 (mean+/-sd) days after the symptom onset. The mean length of hospital stay for non-cancer cases were 13.6+/-7.27 (mean+/-sd), and 16.11+/-8.69 (mean+/sd) before the individuals were either recovered, discharged or deceased (Figs. 2B, 2C). The mean serial interval of the fitted normal distribution is 5.09 (95\% Cl: 4.87-6.07) days for non-cancer patients, while it was 3.69 (95\% Cl:3.02-4.39) days for cancer cases (Fig. 3A, 3B, 3C; supplementary tables s3; s4, and s6; Supplementary Fig. S2).

\section{SARS-CoV-2 viral load is higher in cancer patients than non-cancer patients}

We report SARS-CoV-2 viral load analysis from two commercial viral detection assay kits (details in Materials and methods section). We analyzed the impact of SARS-CoV-2 viral load for all 64 cancer patients as well as 120 non-cancer patients with confirmed Covid-19 diagnosis. All patients were tested using nasopharyngeal swab. To investigate the impact of SARS-CoV-2 viral load, we have obtained the Ct values for SARS-CoV-2 specific gene target quantified using two separate assay which specifically target SARS-CoV-2. We first compared the results with that of Roche cobas SARS-CoV-2 ORF-1ab and E gene. Results show that MOLgen-SARS-CoV-2 and Sansure Biotech SARS-CoV-2 N gene is highly correlated with that of the Roche cobas ORF-1 $\mathrm{ab}$ and $\mathrm{E}$ genes and no significant $\mathrm{Ct}$ value variations were found among the two detection kits (Supplementary Fig. S3). The only differences between the two assay kits was data generated from MOLgen SARS-CoV-2 detection was 2 cycle higher than the Ct values obtained from Sansure SARS-CoV-2 detection kit for N2 gene target for the cobas Ct values ORF-1 ab target.

We transformed the $\mathrm{Ct}$ value into quantitative assessment of viral load. For this, $\mathrm{Ct}$ values were divided into three quartiles Q1, Q2 and Q3. Q1 represents the high viral load (Ct values less than 21.5; and viral load 5.30.1-5.778 $\log _{10}$ RNA copies/mL), Q2 medium viral load (Ct value between 21.5-27.3; 3.699-5.30 $\log _{10}$ RNA copies $/ \mathrm{mL}$ ) and low viral load (Ct values over 27.3; $2.67-3.69 \log _{10}$ RNA copies $/ \mathrm{mL}$ ). The median Ct values for the SARS-CoV-2-specific gene target for cancer (MOLgen detection assay) was 21.5 (IQR-17.3-29.57) compared with a median Ct values of over 23.7 (IQR $=22.24-32.4)$ in non-cancer patients (Fig. 4A). Patients age has shown the significant positive correlation with high viral load (Fig. 4B, $\left.p<0.0001 ; R^{2}=0.50\right)$ ), but there was no significant difference in viral load between male and female (Fig. $4 \mathrm{C}, \mathrm{p}=0.32$ ). 
Using this cut-off, compared with Covid-19 patients without cancer and low viral load, cancer patients and high viral load had higher observed severe patient's condition (OR,1.42; 95\% Cl 1.17-3.003; $\mathrm{p}<$ 0.0001 ), higher death (OR, 1.62; 95\% $\mathrm{Cl} 1.27-3.25 ; \mathrm{p}=0.00016)$; ICU admission (OR 1.25; $95 \% \mathrm{Cl} 1.07-$ $2.81, p=0.00029)$ and higher chances of requiring IVS (OR, 1.10; 95\% $\mathrm{Cl} 0.91-2.38 ; \mathrm{p}=0.098$; Fig. 4D; supplementary table s7). We then analyzed the possible occurrence of severity, death, ICU admission and IVS requirement in cancer and non-cancer patients' group by cumulative survival analysis. We found that the severe condition occurred earlier in cancer patients where SARS-CoV-2 viral load is higher than low SARS-Cov-2 viral load in non-cancer patients (Figs. 4E; 4F). These results are consistent with results obtained for multi-variate regression results after adjusting for patients underlying medical conditions (supplementary table s1a; s1b and s1c).

\section{High viral load is associated with severe condition and high mortality in certain type of cancer patients}

Next, we sought to compare SARS-CoV-2 viral load and patient's severity and mortality in several cancer type and non-cancer cohort. The mortality rate was $41.38 \%$ for high viral load; $23.81 \%$ in medium viral load and $14.29 \%$ in low viral load cancer patient, while the mortality was lower in non-cancer patients (22.22\% for high viral load, $5.13 \%$ in medium viral load and $1.85 \%$ in low viral load patients; Table 2 ). We then compared viral load analysis between the different type of cancers and non-cancer patients using similar cutoff criteria above. As shown in Figs. 5A; 5B, lung, colon, hematologic, esophageal and breast cancer presented high SARS-CoV-2 viral load with higher risk of severe patients' conditions, have relatively high death rate, ICU admission and comparatively higher chance of requiring IVS in the shortest possible time.

Table 2

Mortality rate of cancer and non-cancer patients based on SARS-CoV-2 viral load

\begin{tabular}{|c|c|}
\hline \multicolumn{2}{|c|}{ Non-cancer patients COVID-19 positive } \\
\hline Viral load & $\%$ Mortality \\
\hline Low $(n=54)$ & $(n=1 ; 1.85 \%)$ \\
\hline Medium $(n=39)$ & $(n=2 ; 5.13 \%)$ \\
\hline High $(n=27)$ & $(n=6 ; 22.22 \%)$ \\
\hline \multicolumn{2}{|c|}{ Cancer patients with COVID-19 positive } \\
\hline Low $(n=14)$ & $(n=2 ; 14.29 \%)$ \\
\hline Medium $(n=21)$ & $(n=5 ; 23.81 \%)$ \\
\hline High (n = 29) & $(n=12 ; 41.38 \%)$ \\
\hline
\end{tabular}


Among the 64 Covid-19 positive cancer patients, patients have received different therapeutic regimens, such as, chemotherapy, surgery or radiotherapy treatments. Thirty-three $(33 / 64 ; 5156 \%)$ had chemotherapy, $5(5 / 64 ; 7.81 \%)$ had radiotherapy, 10 (10/64; 15.63\%) had surgery and 16 (16/64; 25.0\%) had surgery before these patients were tested positive for Covid-19 (supplementary table s8). Patients who received chemotherapy tended to show high rate of developing severe condition in patients with high SARS-CoV-2 viral load compared to low viral load (Fig. 5C; 5D). However, radiotherapy and surgery had medium to no effects in developing severs condition with lower low viral load. When mortality/death was assessed, chemotherapy, surgery and radiotherapy treated patients having high viral load and lead to higher rate of death, higher chances of ICU admission and higher use of IVS (Fig. 5C; 5D). Additionally, the relationship between patients who received chemotherapy and underlying conditions and disease severity as well as disease outcome for Covid- 19 positive cases shown in Fig. 5E and 5F.

\section{Discussion}

Our study demonstrated that the differences in SARS-CoV-2 viral load in Covid-19 positive cancer and non- cancer patients may play a role in the prediction of mortality and the extent of disease severity. SARS-CoV-2 viral load is significantly higher in cancer patients with increasing disease severity and mortality compared to non-cancer patients. Several phenomenal observations have emerged from our study. First, cancer patients with active Covid-19 infection showed shorter incubation period and serial interval time when compared with non-cancer patients. Secondly, patients with cancer infected with Covid-19 and high viral load is more likely to experience severe and deleterious outcomes compared to patients with non-cancer and low viral load. Thirdly, lung and breast cancer patients with high viral load demonstrated higher gravity of severe events, i.e death, ICU support, invasive ventilation requirement compared with non-cancer and low viral load individuals. Lastly, cancer patients who are under active anti-cancer treatment or have been previously treated with anticancer agents, particularly chemotherapeutic treatment showed higher death rate and higher chances of experiencing critical symptoms due to high SARS-CoV-2 viral load. These results may be useful for the consideration of prognostic tools to monitor and stratify the patients for delivering relevant treatment.

Since the emergence of Covid-19, the virus has rapidly spread all over the world and many countries are grappling for the search of epidemiological characteristics and control of the transmission of the virus, including its assessment of overall outcome and its impact in the society. An astonishing feature of Covid-19 pandemic is that elderly male reported severe disease and higher mortality than females ${ }^{15-19}$. Moreover, age, smoking status, and comorbidities, such as, hypertension and cardiovascular disease are the risk factors for severe disease and mortality among patients with cancer and non-cancer Covid-19 patients ${ }^{8,20}$. Since the start of the global pandemic of Covid-19, patients with cancer in most countries became the center point of concern due the highly vulnerability, increased risk of contracting Covid-19, severe outcomes and comparatively higher rate of requiring intensive care and increased risk of death 6,21. Given the similar epidemiological features between cancer and non-cancer Covid-19 patients, it is highly likely that cancer patients endow some additional features different from non-cancer individuals. 
There are considerably wide gaps remains in our understanding of SARS-CoV-2 pathogenesis, including whether levels of viral load and disease severity in patients with cancer. To ascertain these additional attributes, this study was conducted to find out the possible effects of SARS-CoV-2 viral load in cancer patients and possible outcomes. Our study evaluating the patient's severe condition and death not only confirm the association of higher-level viral load with these factors, but these factors have an additive effect which may contribute to the increased risk of mortality and severe condition in patients with multiple risk factors.

Perhaps the most important observation in our study is that, Covid-19 patients with cancer and high viral load had significantly more severe outcomes, higher rates and longer stay in the hospitals and ICU and invasive ventilation supports compared to low viral load and non-cancer patients, confirming fraction of previously published reports ${ }^{6,22}$. Outcome of Covid-19 positive patients without cancer was characterized by relatively lower death rate $(2-3 \%)^{22}$, in contrast the rate of death in Covid-19 positive cancer patients exceeded considerably (3-folds), suggesting the vulnerability of cancer patients during pandemic ${ }^{22}$. In our study we did observe a substantial impact of high SARS-CoV-2 viral load on the cancer patient's death rate, disease severity, which may suggest that distinct factors modulating Covid-19 susceptibility and related outcome to the cancer patients ${ }^{2,22,23}$.

Outcome of Covid-19 positive non-cancer and cancer patients was also characterized by patients' underlying conditions. Patients experiencing one or more underlying condition are likely to exhibit a more severe form of Covid-19 symptoms. In a large cohort study $(n=22,753)$, it was reported that, major underlying conditions were cardiovascular disease (8.9\%), hypertension $27.4 \%)$, diabetes (17.4\%) and cancer (3.5\%, [ref: 21]). This suggests that presence of any of these underlying conditions and high viral load may severely affect the clinical outcome of patients with Covid-19 ${ }^{25}$. Our findings are consistent with those presented in the previous studies suggesting that potentially additive adverse effects of one or more underlying conditions of Covid-19 positive cancer patients with high SARS-CoV-2 viral load.

We described a cohort of cancer patients with confirmed Covid-19 positivity and explored the association of viral load and outcome of cytotoxic chemotherapy. Our cohort includes breast (25.0\%), lung (14.06\%), head and neck $(9.38 \%)$, bladder $(9.38 \%)$, colon $(10.94 \%)$, and ovarian $(7.81 \%)$ cancer. We identified that all cancer patients with high viral load experienced the severe symptom of Covid-19 with the lifethreatening possibility and deaths. A substantial proportion of death was observed in severe and critical cases. The mortality rate is higher than the low viral load non-cancer patient's cohort which may characterize the severity of the symptoms of cancer patients with high viral load. The rate of hospitalization and seeking ICU and invasive ventilation support was much higher than those of noncancer patients which was approximately $29.41 \%$ vs $1.67 \%$ and $17.65 \%$ vs $0.0 \%$ respectively. This finding thus suggests that having a detection of Covid-19 on cancer patients with high viral load substantially increases the burden of hospitalization and utilization of more intensive care supports. From our analysis, it was observed that patient's with lung cancer had a higher death rate followed by breast cancer among all patients with cancer. The average time to death was shorter, and had a rapidly 
deteriorating clinical condition once tested positive for Covid-19 who upon admission to hospital detected high SARS-CoV-2 viral load. This adverse clinical condition may be due to high viral load for most cancer patients. We speculate that compromised immune systems due to cytotoxic chemotherapy may play a role in impeding the immunologic functions and thereby deteriorating clinical conditions for cancer patient's death. Although our small sample size limits us to draw a definitive conclusion about our observation, it could be used as a reference point along with other larger cohort studies $2,22,23,26,27$. A recent report showed that patients with hematologic and metastatic lung cancer had the highest frequency of severe events ${ }^{22}$. Our results are consistent with this report at least for lung cancer, since cancer cohort is smaller in hematologic malignancy cases thus limiting our understanding of severe events on hematologic cancer.

In our cohort, patients with cancer mainly received two major cancer related treatments, and therapies including chemotherapy and radiotherapy. Outcome of cancer patient's treatment tested positive for Covid-19 was characterized by higher rate of death and severity. Patients receiving chemotherapy within 4-6 months before confirmed Covid-19 infection had the highest rate of mortality and severity of illness, which is strikingly contrasting to recently published results ${ }^{23}$. Several recent studies reported that hematological malignancies and patients receiving immunotherapies had the highest death rate and severe clinical phenotypes $2,22,23$. Our study is lacking patients who receive targeted therapy, immunotherapy and we cannot conclude that only cytotoxic chemotherapy is solely responsible for higher mortality and severe patients' characteristics. A larger cohort with a variety of treatment strategies may precisely answer our observations.

Finally, we analyzed the serial interval and incubation period differences between non-cancer and cancer cohorts, which are key parameters for transmission modeling and assists governments and policy makers to respond to the pandemic. These two parameters influence the disease incidence and prevalence of transmission ${ }^{12}$. In the non-cancer and cancer cohort, we estimated the serial interval, and obtained a shorter serial interval (3.9 days) for cancer patients' cohort, while the non-cancer patients serial interval was 5.09 days ${ }^{12}$. Furthermore, the estimated serial interval is shorter than the incubation period in cancer cohorts suggesting that the pre-symptomatic transmission for cancer patients. The striking observation was that the source of Covid-19 infection in most cancer patients was from hospital facilities probably due to the frequent treatment visits during the pandemic. This study thus suggests that the hospital related infection could be avoided by delaying or cancelling cancer treatment if possible.

As usual our study carries several limitations. Although our study cohort for cancer is comparatively smaller than recently published studies and only symptomatic cases were included who seeking help from health care providers, but larger cohort as well as asymptomatic cases, and hidden cases who did not report for assistance could further extends our understandings of actual characteristics of cancer patients' treatment, and Covid-19 infection. Furthermore, due to the government pandemic restrictions it was not possible to collect all the available patient's data and records from the health care providers which potentially impeded our analysis. With some larger number of patient's analysis would be able 
pinpoint more unanswered questions. Despite all these limitations, our study is unique when compared with the larger studies, representative of small numbers of cancer patients among hundreds of unidentified cases. Future work with diverse cancer type and larger patients' cohort and long- term followups will define the specific risk of Covid-19 on outcomes in much greater gravity in patients with cancer.

\section{Declarations}

\section{Ethics declaration}

\section{Conflict of interest}

The authors declared no conflict of interest

\section{Acknowledgements}

The authors express sincere thanks to all patients and all the oncology physicians and hospital personnel to assist retrieving the patient's data during pandemic.

\section{The role of funding source}

This study was supported by Bangladesh Medical Research Council and King Faisal Specialist Hospital and Research Centre internal grant. The funding agency had no roles in study design, data collection and analysis, data interpretation and writing of the manuscript

\section{Author information}

These authors contributed equally: Maha Al-Mozaini, Abu Shadat M Noman

\section{Affiliations}

Department of Infection and Immunity, King Faisal Specialist Hospital and Research Centre, Riyadh, Saudi Arabia

Maha Al-Mozaini

Bangamata National Cellular and Molecular Research Centre, Bangladesh

Abu Shadat M Noman

Department of Medicine, King Faisal Specialist Hospital and Research Centre Jawaher Alotaibi

\section{Department of Medicine, Park View Hospital, Chittagong, Bangladesh}

Mohammed Rezaul Karim, ASM Zahed, ATM Rezaul Karim 
Clinical Genomic Centre, King Faisal Specialist Hospital and Research Centre, Riyadh, Saudi Arabia

Khaldoun Alromiah ${ }^{5}$

Department of Molecular Oncology, King Faisal Specialist Hospital and Research Centre, Riyadh, Saudi Arabia. School of Medicine, Al-Faisal University, Riyadh, Saudi Arabia

Syed S Islam

\section{Authors contribution}

SSI have contributed to the design of the study, SSI and MA contributed in data analysis, SSI, MA and ASMN contributed in writing the manuscript. SSI, ASMN, KA, MA, JA, ATMRK, ASMZ, MRK have contributed to literature search, data collection, data interpretation, and clinical data inconsistencies. All authors reviewed the manuscript and have given final approval of the submitted version.

\section{Data availability}

The data that supports our results of this study are available upon request from the corresponding author.

\section{Informed consent statement}

This work was conducted as part of the ongoing public health response, therefore institutional or regional review board approval and individual cases consent approval was waived. This study was approved by ethics committee of the Bangladesh Medical Research Council (BMDC) and King Faisal Specialist Hospital and Research Centre.

\section{References}

1. WHO \& Aylward, Bruce (WHO); Liang, W. (PRC). Report of the WHO-China Joint Mission on Coronavirus Disease 2019 (COVID-19). WHO-China Jt. Mission Coronavirus Dis. 2019 (2020).

2. Kuderer, N. M. et al. Clinical impact of COVID-19 on patients with cancer (CCC19): a cohort study. Lancet (2020) doi:10.1016/S0140-6736(20)31187-9.

3. Mehta, V. et al. Case fatality rate of cancer patients with COVID-19 in a New York Hospital system. Cancer Discov. (2020) doi:10.1158/2159-8290.CD-20-0516.

4. Yu, J., Ouyang, W., Chua, M. L. K. \& Xie, C. SARS-CoV-2 Transmission in Patients with Cancer at a Tertiary Care Hospital in Wuhan, China. JAMA Oncology (2020) doi:10.1001/jamaoncol.2020.0980.

5. Miyashita, H. et al. Do patients with cancer have a poorer prognosis of COVID-19? An experience in New York City. Annals of Oncology (2020) doi:10.1016/j.annonc.2020.04.006.

6. Liang, W. et al. Cancer patients in SARS-CoV-2 infection: a nationwide analysis in China. The Lancet Oncology (2020) doi:10.1016/S1470-2045(20)30096-6. 
7. Magleby, R. et al. Impact of Severe Acute Respiratory Syndrome Coronavirus 2 Viral Load on Risk of Intubation and Mortality Among Hospitalized Patients With Coronavirus Disease 2019. Clin. Infect. Dis. (2020) doi:10.1093/cid/ciaa851.

8. Westblade, L. F. et al. SARS-CoV-2 Viral Load Predicts Mortality in Patients with and without Cancer Who Are Hospitalized with COVID-19. Cancer Cell (2020) doi:10.1016/j.ccell.2020.09.007.

9. Fajnzylber, J. et al. SARS-CoV-2 viral load is associated with increased disease severity and mortality. Nat. Commun. (2020) doi:10.1038/s41467-020-19057-5.

10. Vogels, C. B. F. et al. Analytical sensitivity and efficiency comparisons of SARS-CoV-2 RT-qPCR primer-probe sets. Nat. Microbiol. (2020) doi:10.1038/s41564-020-0761-6.

11. Silva, J. et al. Saliva viral load is a dynamic unifying correlate of COVID-19 severity and mortality. medRxiv Prepr. Serv. Heal. Sci. (2021) doi:10.1101/2021.01.04.21249236.

12. Tindale, L. C. et al. Evidence for transmission of covid-19 prior to symptom onset. Elife (2020) doi:10.7554/eLife.57149.

13. Adam, D. C. et al. Clustering and superspreading potential of SARS-CoV-2 infections in Hong Kong. Nat. Med. (2020) doi:10.1038/s41591-020-1092-0.

14. McAloon, C. et al. Incubation period of COVID-19: A rapid systematic review and meta-analysis of observational research. BMJ Open (2020) doi:10.1136/bmjopen-2020-039652.

15. Scully, E. P., Haverfield, J., Ursin, R. L., Tannenbaum, C. \& Klein, S. L. Considering how biological sex impacts immune responses and COVID-19 outcomes. Nat. Rev. Immunol. (2020) doi:10.1038/s41577-020-0348-8.

16. Richardson, S. et al. Presenting Characteristics, Comorbidities, and Outcomes among 5700 Patients Hospitalized with COVID-19 in the New York City Area. JAMA - J. Am. Med. Assoc. (2020) doi:10.1001/jama.2020.6775.

17. Singh, S., Chowdhry, M., Chatterjee, A. \& Khan, A. Gender-Based Disparities in COVID-19: Clinical Characteristics and Propensity-matched Analysis of Outcomes. medRxiv (2020).

18. Kragholm, K. et al. Association Between Male Sex and Outcomes of Coronavirus Disease 2019 (COVID-19)-A Danish Nationwide, Register-based Study. Clin. Infect. Dis. (2020) doi:10.1093/cid/ciaa924.

19. Jin, J. M. et al. Gender Differences in Patients With COVID-19: Focus on Severity and Mortality. Front. Public Heal. (2020) doi:10.3389/fpubh.2020.00152.

20. Ye, C. et al. Impact of comorbidities on patients with COVID-19: A large retrospective study in Zhejiang, China. J. Med. Virol. (2020) doi:10.1002/jmv.26183.

21. Curigliano, G. et al. Managing cancer patients during the COVID-19 pandemic: an ESMO multidisciplinary expert consensus. Ann. Oncol. (2020) doi:10.1016/j.annonc.2020.07.010.

22. Dai, M. et al. Patients with cancer appear more vulnerable to SARS-CoV-2: A multicenter study during the COVID-19 outbreak. Cancer Discov. (2020) doi:10.1158/2159-8290.CD-20-0422. 
23. Lee, L. Y. W. et al. COVID-19 mortality in patients with cancer on chemotherapy or other anticancer treatments: A prospective cohort study. Lancet (2020) doi:10.1016/S0140-6736(20)31173-9.

24. Bajgain, K. T., Badal, S., Bajgain, B. B. \& Santana, M. J. Prevalence of comorbidities among individuals with COVID-19: A rapid review of current literature. American Journal of Infection Control (2020) doi:10.1016/j.ajic.2020.06.213.

25. Guan, W. et al. Clinical Characteristics of Coronavirus Disease 2019 in China. N. Engl. J. Med. (2020) doi:10.1056/nejmoa2002032.

26. Lee, L. Y. W. et al. COVID-19 prevalence and mortality in patients with cancer and the effect of primary tumour subtype and patient demographics: a prospective cohort study. Lancet Oncol. (2020) doi:10.1016/S1470-2045(20)30442-3.

27. Fillmore, N. R. et al. Prevalence and Outcome of COVID-19 Infection in Cancer Patients: A National Veterans Affairs Study. JNCI J. Natl. Cancer Inst. (2020) doi:10.1093/jnci/djaa159.

\section{Figures}




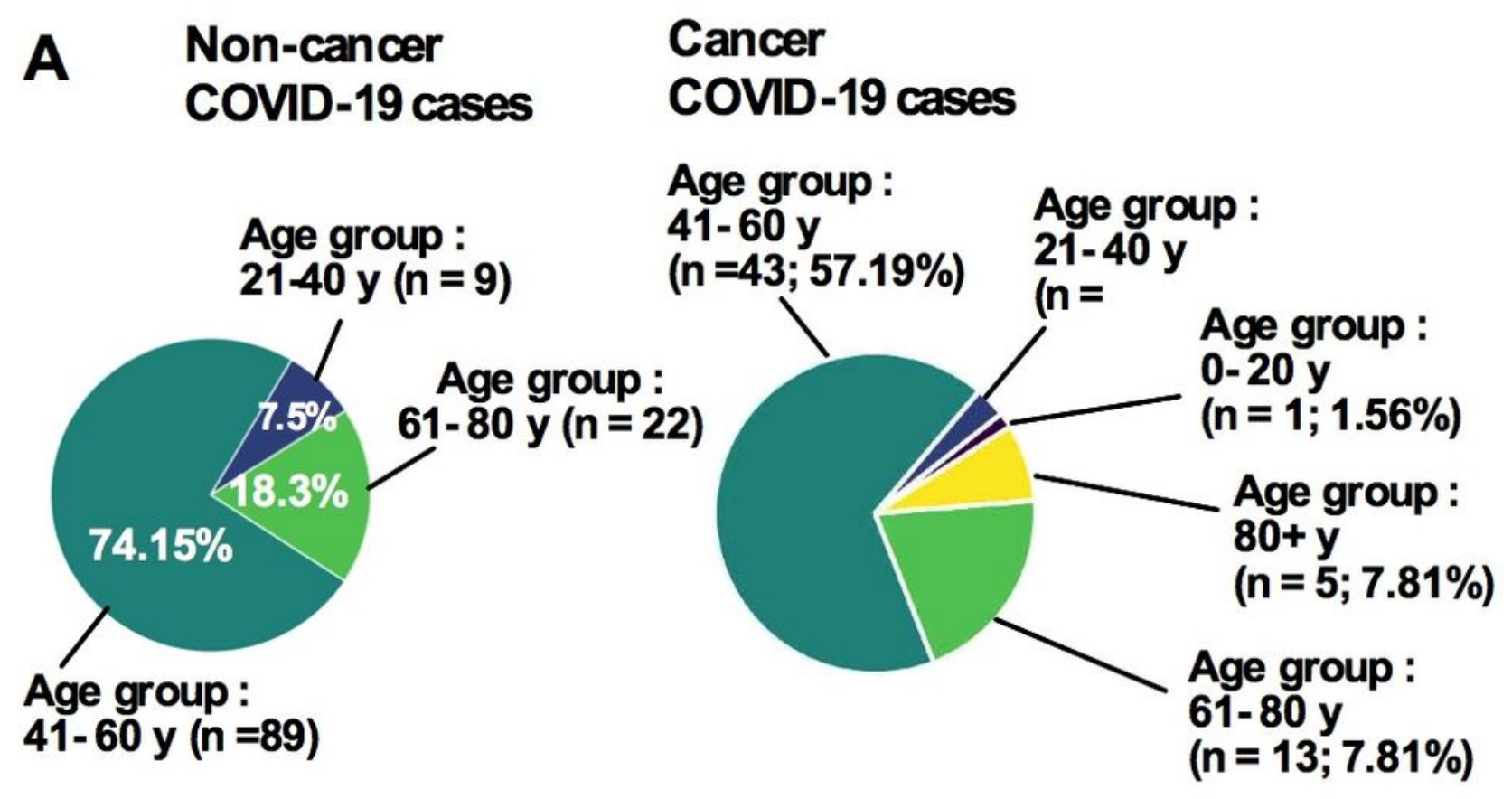

B Incidence of severe condition amomg cancer and non-cancer patients

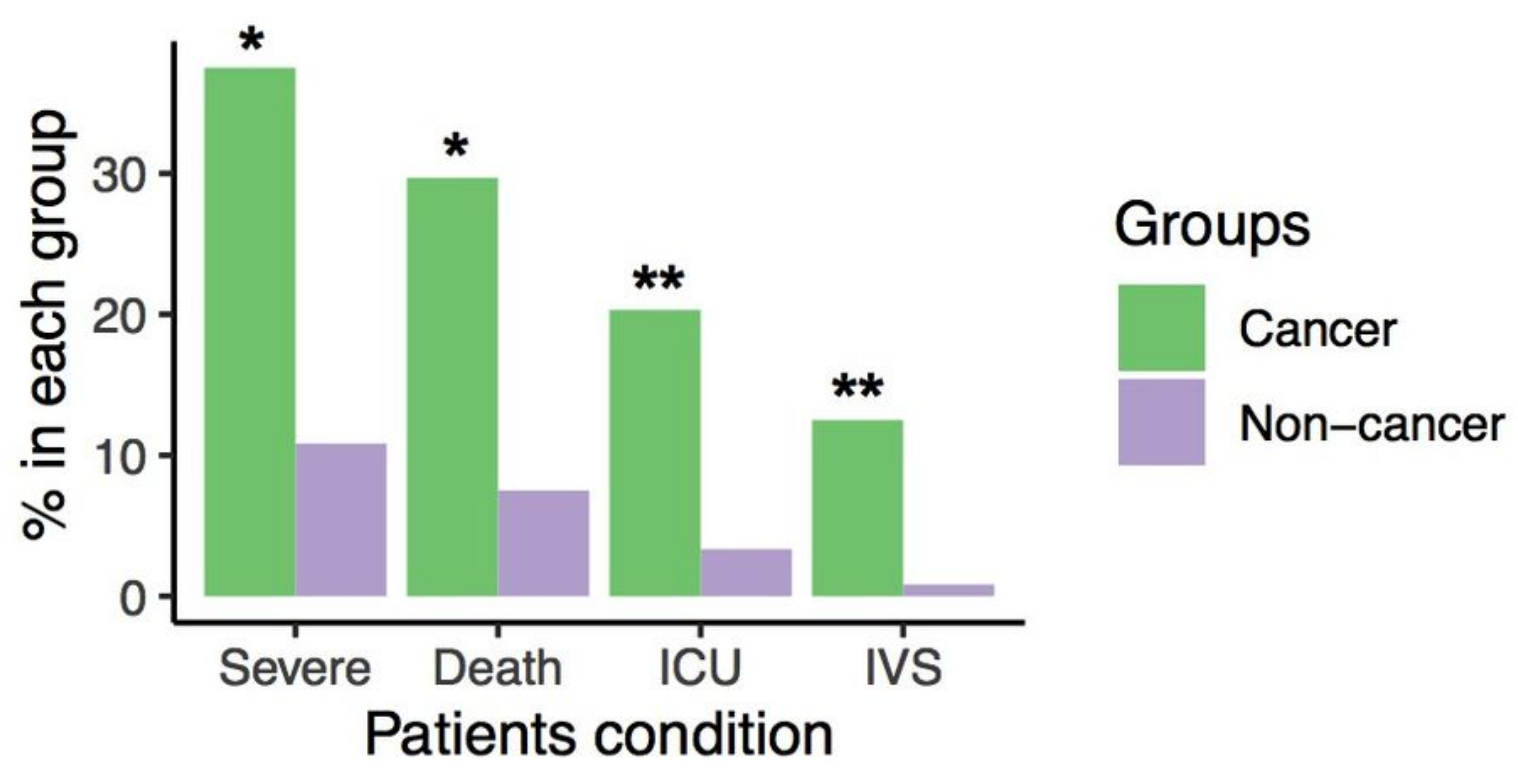

Figure 1

Clinical characteristics of patients among non-cancer and cancer patients. A. Age distribution of patients with non-cancer and cancer patients. B. Incidence of severe condition among non-cancer and cancer patients. 


\section{A \% of COVID-19 infected cases \\ colored by cancer types}

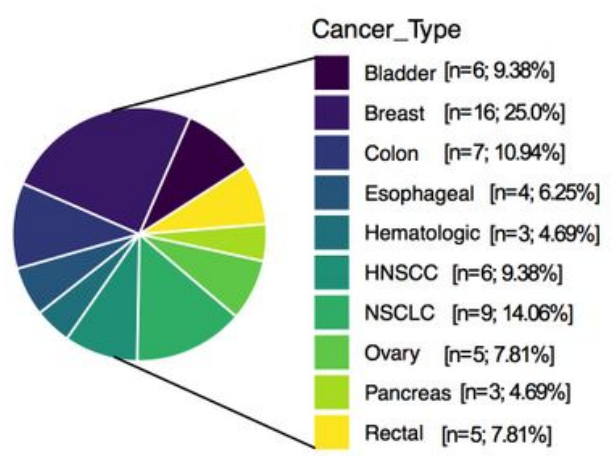

B

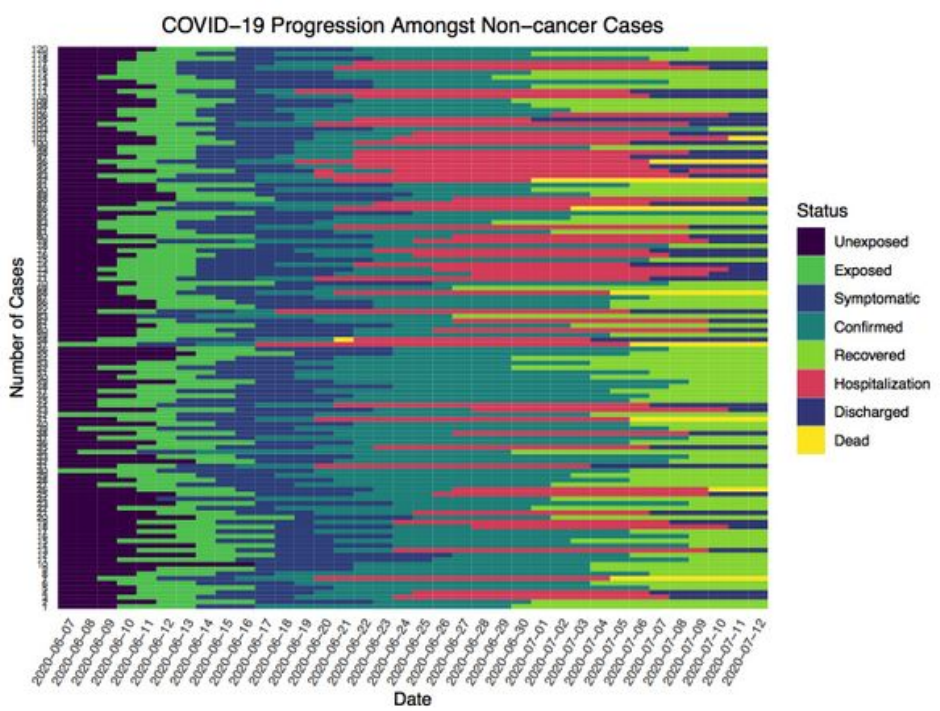

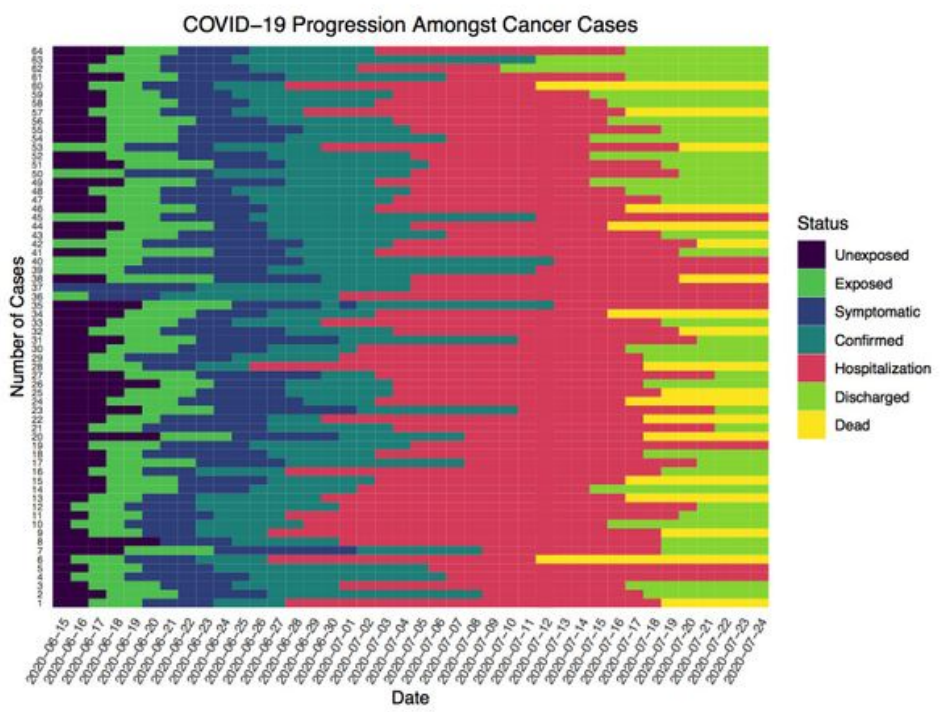

Figure 2

A. Percentage of Covid-19 positive cancer cases colored by cancer types. B and C. Disease timelines: date at which cases is unexposed, exposed, symptomatic, date of confirmed, recovered, date of hospitalization, discharged and deaths of cancer and non-cancer Covid-19 cases. 


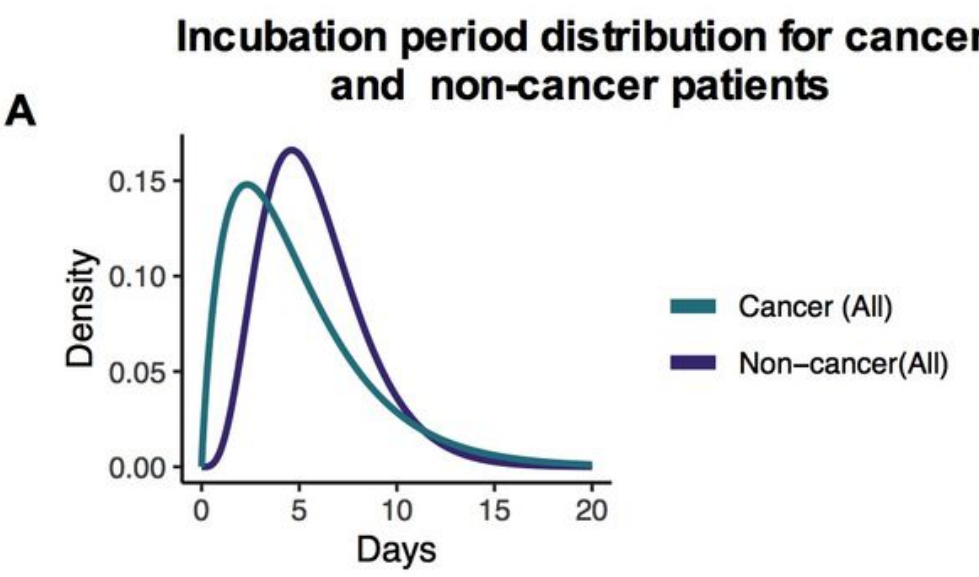

B COVID-19 positive non-cancer patients

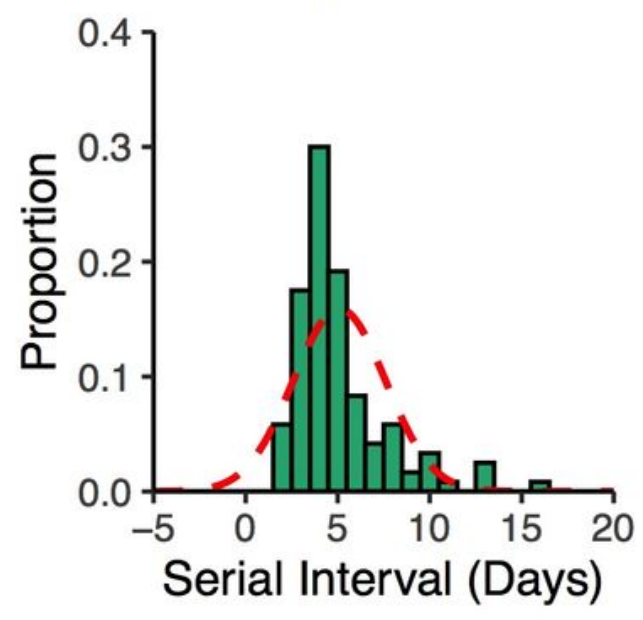

C COVID-19 positive cancer patients

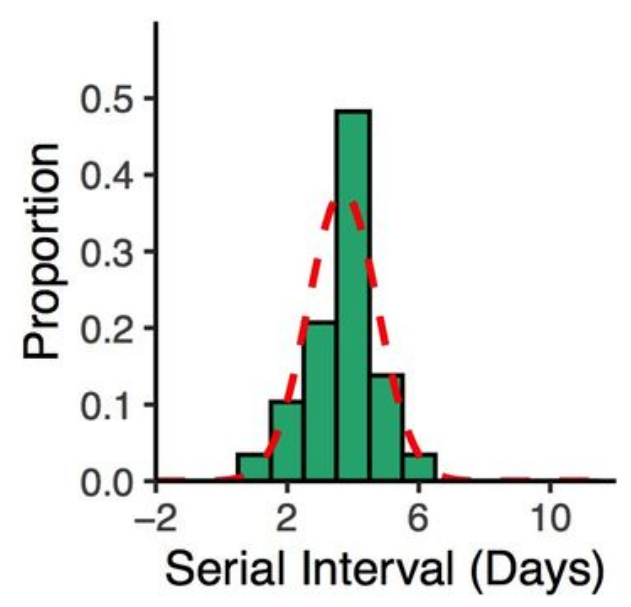

Figure 3

A. Fitted gamma Covid-19 incubation period distributions for all non-cancer and cancer cases including cancer specific cases. B and C. Serial interval distribution of Covid-19 infection cases among cancer and non-cancer cases. 


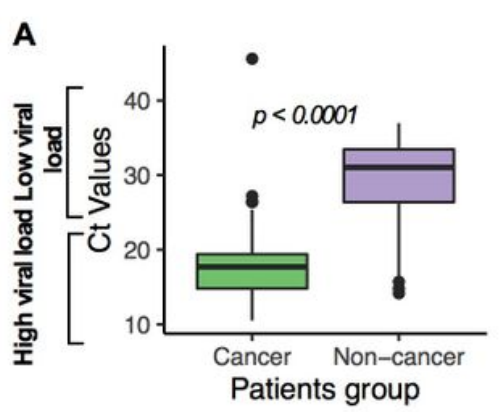

D

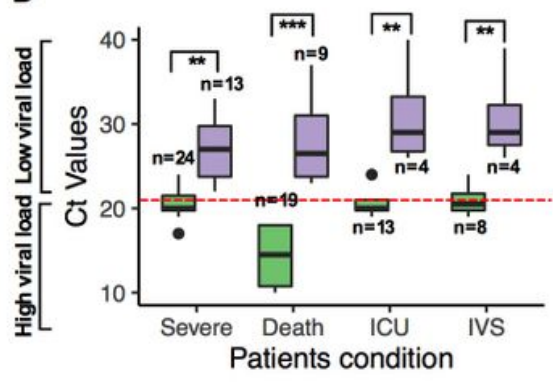

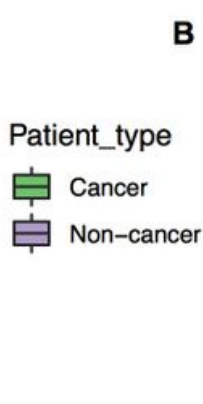

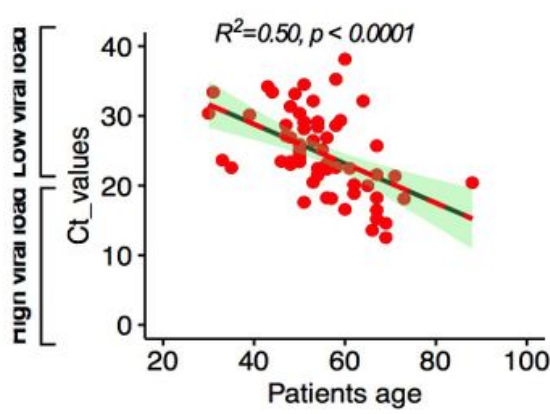

E

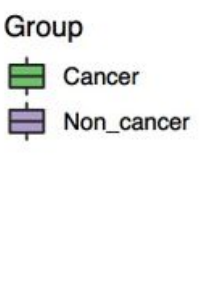

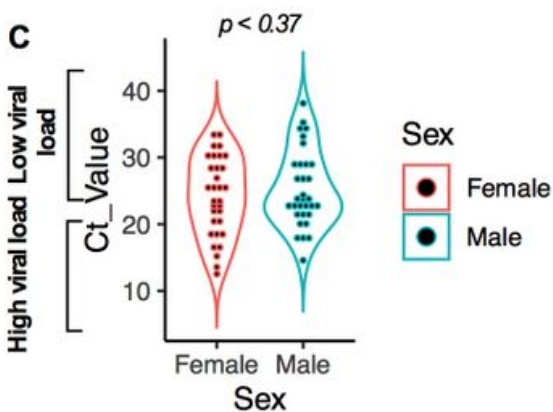
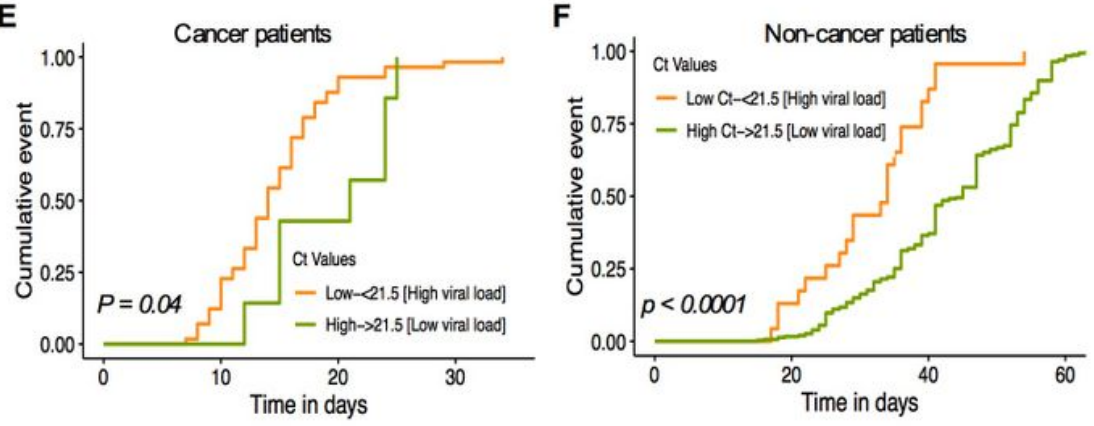

Figure 4

A. SARS-CoV-2 Ct values in cancer and non-cancer patients derived from nasopharyngeal swab test. B. Linear regression of age and viral load. C. t-test comparing the viral load among male and female cases. D, E and F. SARS-CoV-2-viral load and patients' severe condition in patients with and without cancer. 
A

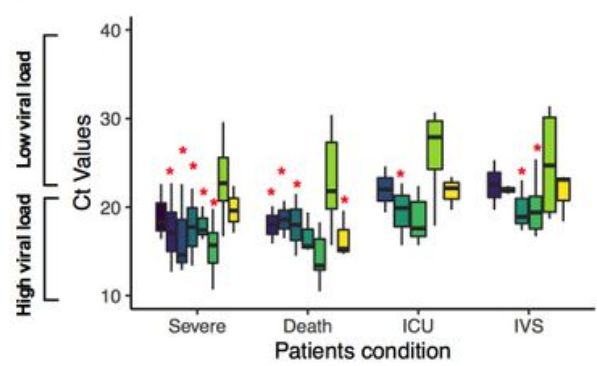

B

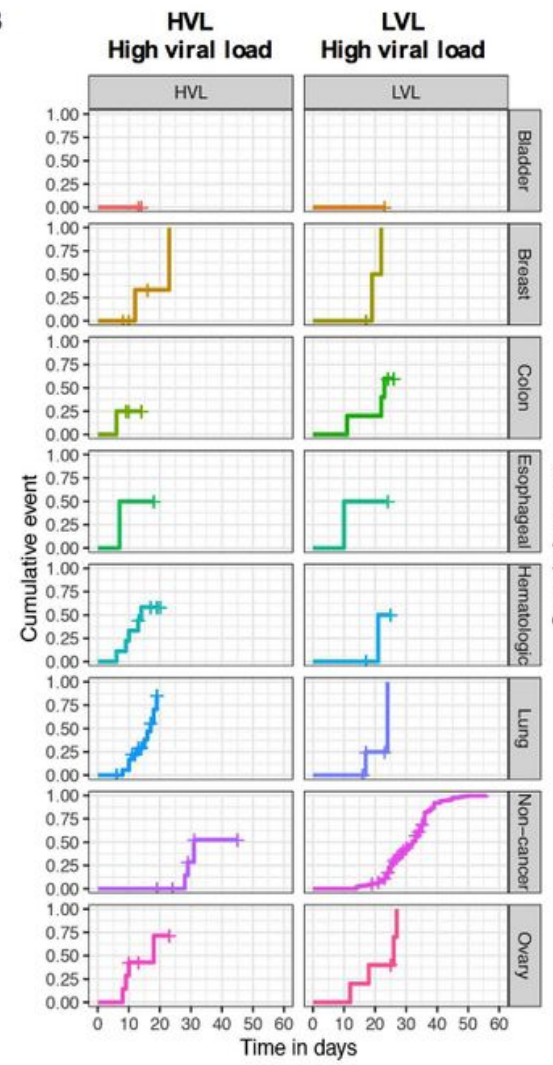

Cancer type

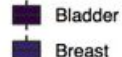

Breast

Colon

峞 Esophageal

Hematologic

宁 Lung

官 Non-cancer

官 Ovary

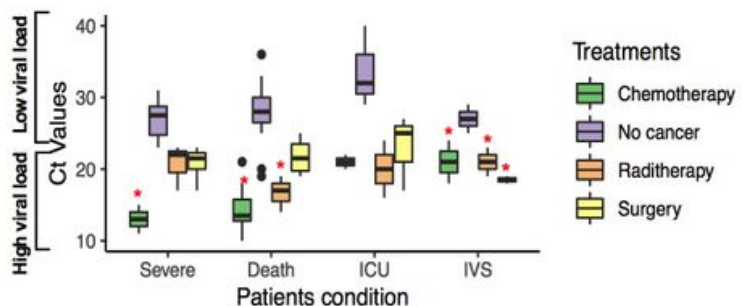

D
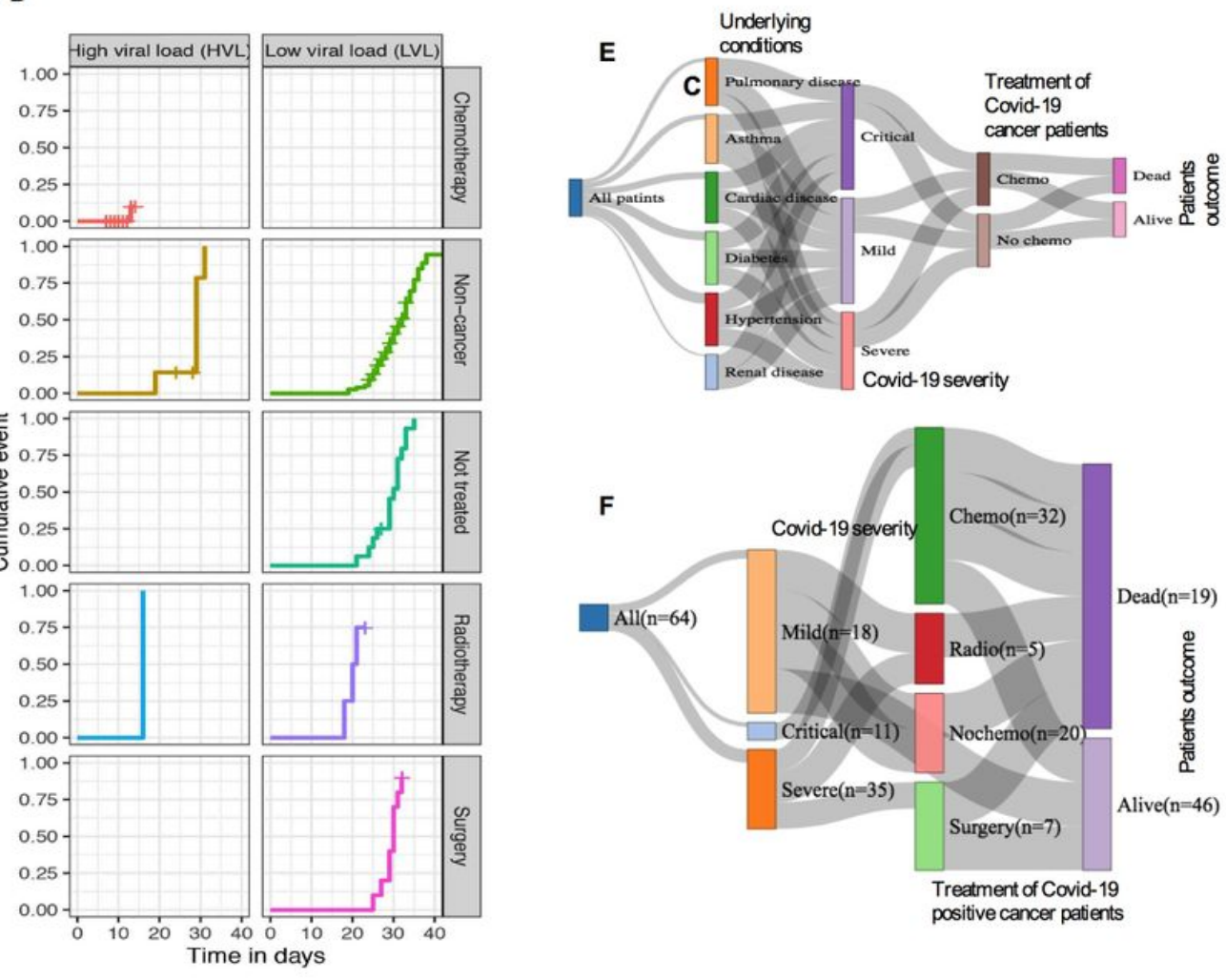

\section{Figure 5}

A and B. SARS-CoV-2 Ct values in different type of cancer patients derived from nasopharyngeal swab test. C and D. Relationship of chemotherapy use of confirmed Covid-19 cases and the mortality and severity of disease outcome. E and F. Relationship of patients underlying condition, disease severity, and chemotherapy treatment and patient's outcome.

\section{Supplementary Files}

This is a list of supplementary files associated with this preprint. Click to download.

- SupplementaryFigS1.tiff

- SupplementaryFigS2.tiff

- SupplementaryTables1as1bs1COddsratios.docx

- SupplementaryTableS2.docx 
- SupplementaryTablesS3S4Incubationperiodandseriallnterval.docx

- SupplementaryTableS5.docx

- SupplementaryTableS6.docx

- SupplementaryTables70ddsratioanalysisforHighviralloadanddifferentvariables.docx

- SupplementaryTableS8Patientscharacteristics.docx

- GraphicalAbstract.tiff 